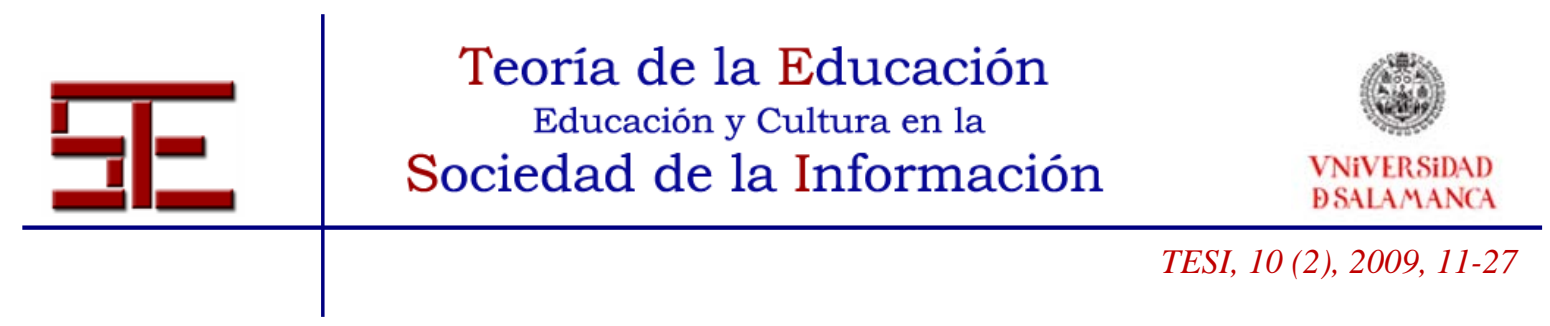

\title{
LA ALFABETIZACIÓN TECNOLÓGICA
}

Resumen: La incorporación a la sociedad del conocimiento es posible mediante la alfabetización tecnológica. Para poder conseguir una educación de calidad a través del uso de las tecnologías es necesario una alfabetización tecnológica entendida como la capacitación no solo instrumental, sino la adquisición de las competencias necesarias para la utilización didáctica de las tecnologías y poder acceder al conocimiento. A través de la alfabetización tecnológica se democratizan los procesos de formación y se consigue la inclusión social, laboral y una mejora en la calidad de vida.

Palabras Claves: Alfabetización tecnológica, formación virtual, inclusión social

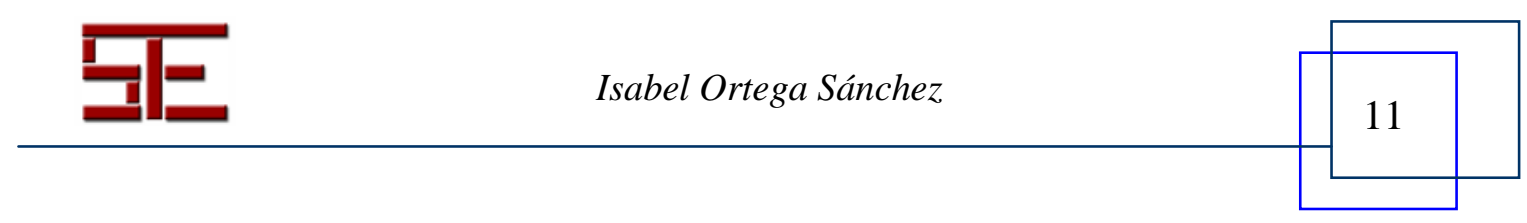




\section{THE TECHNOLOGICAL LITERACY}

Summary: The incorporation to the society of the knowledge is possible through the technological literacy. In order to to achieve a quality education through the use of the technologies is necessary a technological literacy understood as the training not only instrument, but the acquisition of the necessary powers for the didactic use of technologies and to be able to to access to the knowledge. Through the technological literacy are democratized formation processes and is achieved the social, work inclusion and an improvement in the quality of life.

Key Words: Technological literacy, virtual formation, social inclusion

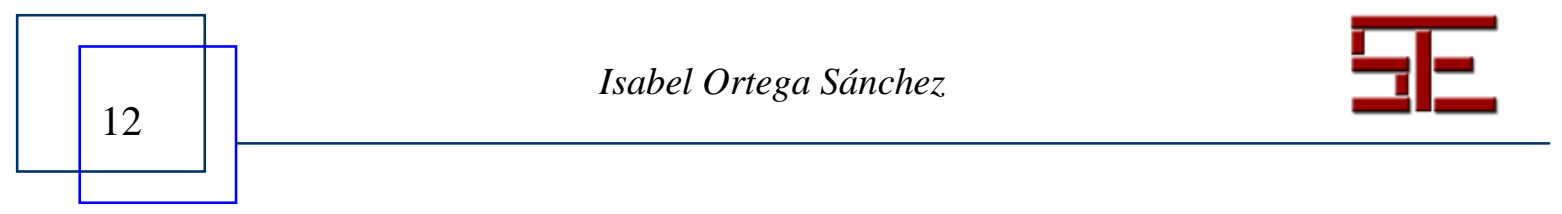




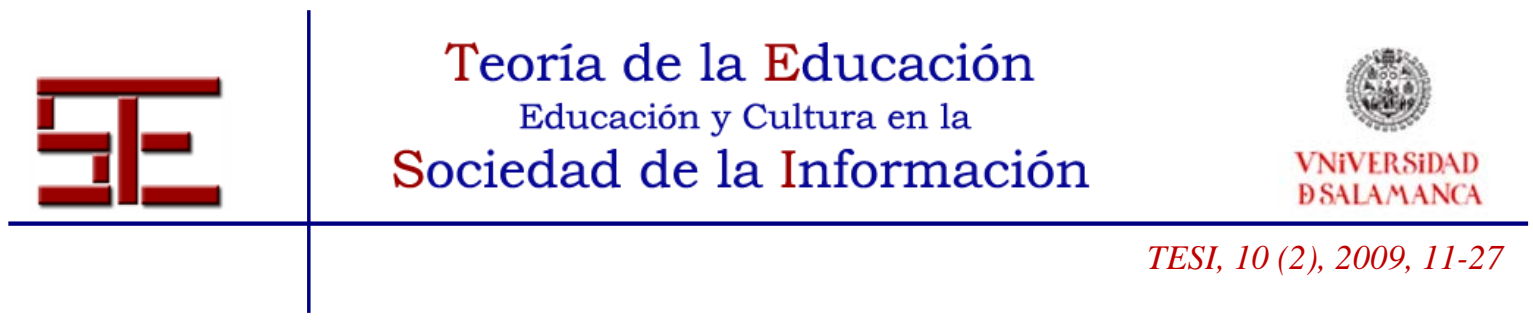

\section{LA ALFABETIZACIÓN TECNOLÓGICA}

Dra. Isabel Ortega Sánchez

iortega@edu.uned.es

Universidad Nacional de Educación a Distancia.

\section{1.- INTRODUCCIÓN}

Las tecnologías son instrumentos para la difusión de la información, la comunicación y la formación, influyendo en todos los contextos políticos, económicos y sociales. El uso de las tecnologías está cambiando las prácticas en el trabajo, construyendo nuevos entornos sociales, laborales, de ocio, cambiando los estilos de vida y las formas de participación social, posibilitando la inclusión social.

A través de los procesos de participación virtual y e-inclusión social se forman ciudadanos más críticos y autónomos generando un desarrollo personal, social y ocupacional y proporcionando una mejora en la calidad de vida.

Las tecnologías de la información y la comunicación están posibilitando las construcción de nuevas culturas, redes sociales a través de las nuevas formas de relacionarse e interaccionar. Su utilización se globaliza mediante nuevos soportes digitales que promueven el e-learning, eJusticia, eAdministración, eSalud, etc.

El uso significativo de las tecnologías favorece una educación permanente y una formación continua en diferentes contextos educativos donde se utilizan las tecnologías como medios facilitadores del aprendizaje.

Para generar una sociedad democrática, superando la brecha digital, entendida como la desigualdad para acceder a las tecnologías y formarse, es necesaria una alfabetización tecnológica que facilite la inserción laboral, social y educativa de todas las personas.

\section{2.- LA ALFABETIZACIÓN TECNOLÓGICA}

Para poder conseguir una enseñanza de calidad a través del uso de las tecnologías es necesaria una alfabetización tecnológica entendida como la capacitación no solo

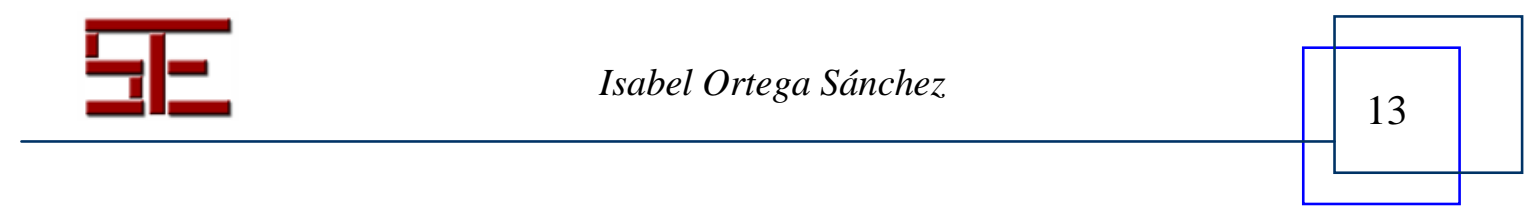




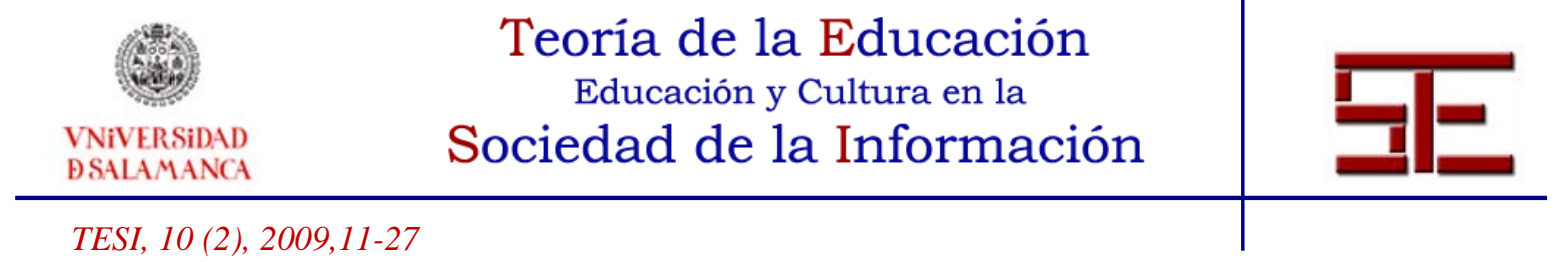

instrumental sino la adquisición de las competencias necesarias para la utilización didáctica de las tecnologías y poder acceder al conocimiento.

Expertos en la utilización de las tecnologías en la educación hablan de alfabetización digital, tecnológica, informacional, etc., resaltando la necesaria capacitación de las personas para acceder al mundo del conocimiento a través de la red.

"La alfabetización digital es el proceso de adquisición de los conocimientos necesarios para conocer y utilizar adecuadamente las infotecnologías y poder responder críticamente a los estímulos y exigencias de un entorno informacional cada vez más complejo, con variedad y multiplicidad de fuentes, medios de comunicación y servicios" Casado

Ortíz (2006: 52)

La alfabetización tecnológica será útil y desarrollará un aprendizaje eficaz y procesos de innovación educativa cuando se genere un cambio en las actitudes, concepciones y prácticas pedagógicas lo que significará modificar el sistema de enseñanza: planificar y contextualizar la formación virtual basada en nuevos principios teóricos y organizativos, usar una metodología contextualizada, desarrollar habilidades socio comunicativa de todas las personas que forman parte de las comunidades educativas o formativas, crear nuevas estrategias de enseñanza-aprendizaje, desarrollar cambios en el papel del docente, crear nuevas figuras mediadoras del aprendizaje, tutores virtuales, cambios del proceso y actividades de aprendizaje del alumnado, cambios en las formas organizativas de las clases, cambios en las modalidades de tutorización, etc.

"La alfabetización no puede simplificarse en el conocimiento y manejo de las destrezas básicas lectoescritoras (hablar, escuchar, leer y escribir) en una sociedad actual. De ahí que sea preciso considerar la alfabetización en medios o audiovisual, ya que los aprendizajes de nuestra época son en clave audiovisual” Aguadez Gómez y Pérez

Rodríguez (2006:69)

Los espacios educativos virtuales se han creado para ofrecer una formación flexible y basada en la libertad práctica que aportan las herramientas de la denominada Web 2.0. Actualmente hablamos de la Web 3.0 que permite realizar asociaciones entre los contenidos de los sitios Web, la transformación de la red en una base de datos, un movimiento hacia hacer los contenidos accesibles, las tecnologías de inteligencia artificial, la Web semántica, o la Web 3D.

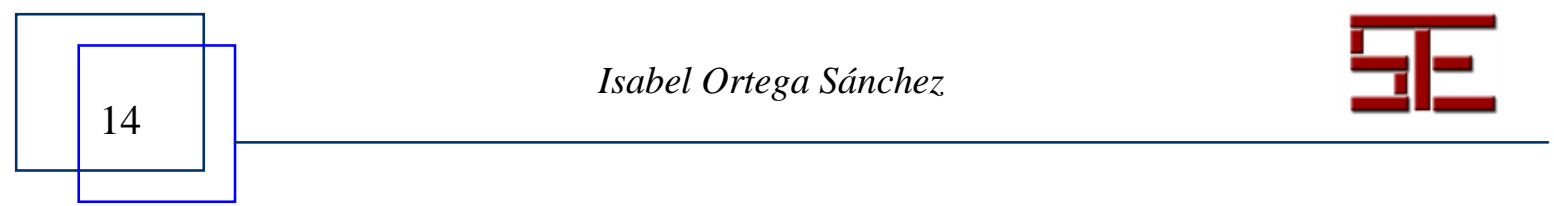




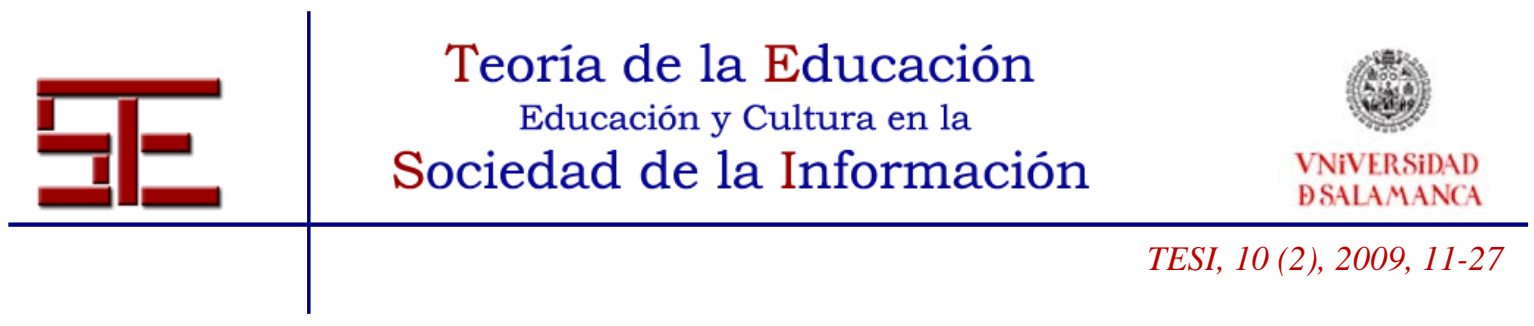

Los nuevos escenarios educativos, en contextos virtuales formales, no formales e informales, abren las puertas a múltiples posibilidades de aprendizaje; aprendizaje autónomo, aprendizaje colaborativo y el desarrollo de competencias para la inserción social y laboral.

En la actualidad aumenta el número de herramientas para la creación y la gestión de contenidos y espacios de formación colaborativos y participativos; Weblog, Wikis, Webquests, Folsksonomías, etc., así como, nuevos dispositivos móviles para el desarrollo de un aprendizaje virtual que potencian las facilidades de acceso, disponibilidad de la información y la portabilidad. El m-learning, el aprendizaje electrónico móvil, facilita el aprendizaje just-in-time a través de los dispositivos portátiles posibilitando una formación virtual de forma inmediata.

Estas nuevas herramientas tecnológicas y sus potencialidades serán aprovechadas si existe una alfabetización tecnológica de toda la comunidad educativa o formativa para poder lograr éxitos en el aprendizaje.

Gutierrez Martín (2006: 60) indica que "la alfabetización globalmente concebida como preparación básica para la vida ha de ser múltiple... útil para la vida que nos ha tocado vivir”. Nos tenemos que adaptar a la formas de comunicación a través de las tecnologías y aprender los nuevos códigos de comunicación, teniendo una visión crítica y reflexiva de las tecnologías.

La formación de los docentes y tutores delimitará el tipo de educación que se genera a través de las tecnologías debido a la importancia del asesoramiento y seguimiento en los cursos virtuales. Una alfabetización tecnológica de toda la comunidad educativa, y especialmente de los formadores, es necesaria si queremos generar espacios de formación virtual de calidad.

En la formación virtual los docentes deben crear objetos de aprendizajes, recursos digitales reutilizables, intuitivos, que promuevan la interacción, sean accesibles y significativos. Los tutores virtuales se centrarán en la dinamización, en el asesoramiento, guía y motivación de los procesos de enseñanza-aprendizaje.

La Unesco (2008) establece los “estándares de competencias en TIC para docentes expresando que "Para vivir, aprender y trabajar con éxito en una sociedad cada vez más

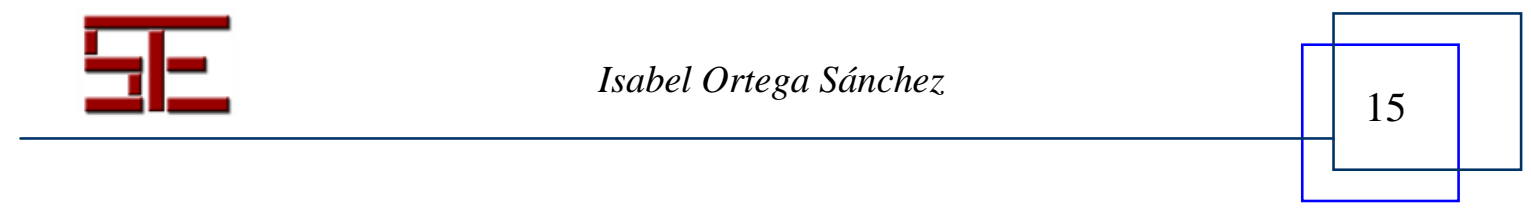




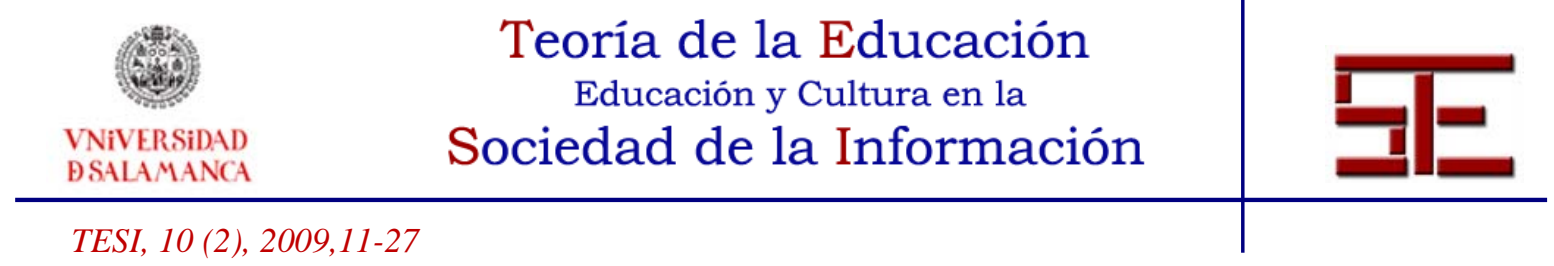

compleja, rica en información y basada en el conocimiento, los estudiantes y los docentes deben utilizar la tecnología digital con eficacia”

La capacitación o alfabetización tecnológica de los docentes, tutores y alumnos debe ser continua, delimitando las competencias y las funciones de cada uno, con el fin de utilizar las tecnologías de forma didáctica y generar espacios virtuales de calidad.

Los alumnos necesitan nuevos contextos formativos que generen competencias a través de la inteligencia colectiva, el conocimiento compartido, la cooperación, la visión crítica, la innovación, la diversidad e interculturalidad y la inteligencia emocional. La adquisición de estas competencias solo será posible a través la alfabetización tecnológica.

"No debemos caer en el error de pensar que las únicas destrezas que deben tener los participantes en las comunidades virtuales son de tipo tecnológicas e instrumentales.

Las comunidades virtuales existen porque hay un objetivo básico de intercambio de in formación y construcción de conocimiento compartido, de ahí que también sean necesarias las habilidades de exposición de los pensamientos, procesamiento de la información, su gestión, comprensión de la información, y síntesis; entre otras” (Cabero Almenara, 2006)

La alfabetización tecnológica promueve la utilización crítica de las tecnologías, así como los conocimientos para aprovechar sus diversas potencialidades pedagógicas, educativas, sociales y comunicativas.

"Un modelo de alfabetización digital va mucho más allá de la simple adquisición de destrezas para utilizar el ordenador o acceder a la red y que por tanto trasciende ampliamente al campo de la informática. Un concepto que requiere habilidades y conocimientos, pero también concienciación y actitudes críticas”. Casado Ortíz (2006:52)

Las desigualdades sociales y la población con riesgo de exclusión social fomentan el acceso desigual al mundo del conocimiento a través de las tecnologías. La alfabetización digital de esta población desfavorecida económica, social y laboralmente es necesaria para desarrollar sociedades más competitivas, mejorar la calidad de vida y favorecer la igualdad de oportunidades.

La educación es la base para el desarrollo económico de un país. Debemos atender a todos los sectores poblacionales para conseguir desarrollos locales y posteriormente globales, centrándonos en la alfabetización tecnológica para acceder a la información, la comunicación y la formación.

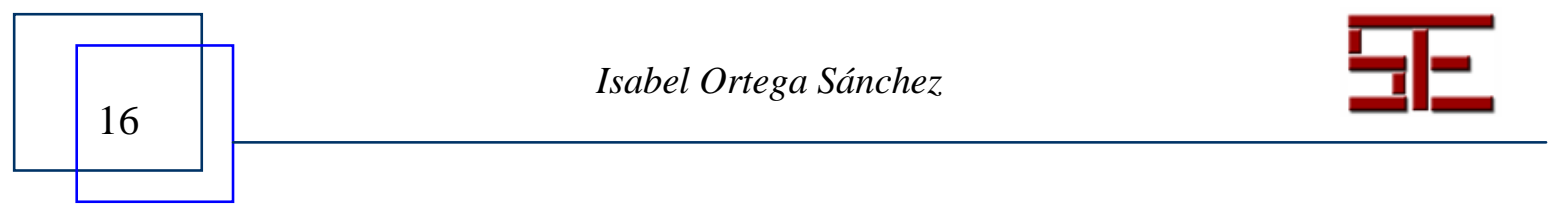




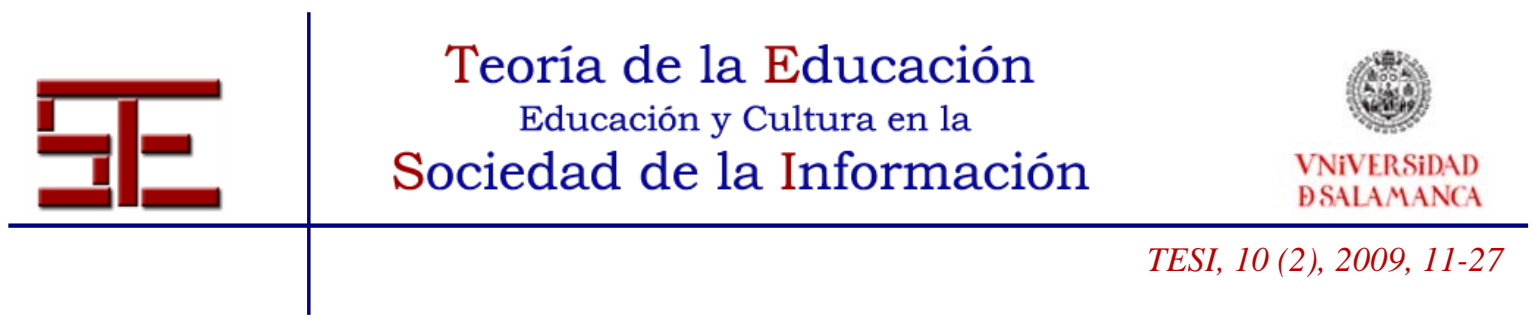

Facilitar el acceso a las tecnologías y la alfabetización digital o tecnológica consigue que los adultos, las personas que viven en zonas rurales y de diferentes clases sociales participen activamente y de manera crítica en una sociedad democrática consiguiendo desarrollar sus potencialidades y generando un capital humano eje del desarrollo integral social.

\section{LA ALFABETIZACIÓN TECNOLÓGICA EN ESPAÑA}

En España se está realizando numerosos proyectos para alfabetizar tecnológicamente a la población, conscientes de la importancia que tiene la educación a través de las tecnologías, así como, la adquisición de las competencias básicas para hacer ciudadanos activos y participativos, generando posibilidades de inclusión social y empleabilidad.

Las líneas de actuación del Plan Nacional de Investigación Científica, Desarrollo Innovación Tecnológica 2008-2011, se inscriben en el Programa Nacional de Reformas. El Programa Nacional de Reformas de España (PNR 2006), que tiene como dos objetivos centrales a alcanzar en el 2010 la convergencia en renta per cápita con la UE y lograr una tasa de empleo del 66\%, se estructura en torno a siete ejes temáticos y en el cuarto eje explica:

Eje 4. I+D+I (Ingenio 2010): destinado al fomento de la I+D+I civil a través de los programas CENIT (colaboración público-privada), AVANZA (impulso e integración de las nuevas tecnologías) Y CONSOLIDER (promoción de la excelencia científica y académica).

En el período 2008-2011 la Acción Estratégica de Telecomunicaciones y Sociedad de la Información deberá responder a los retos planteados por la Sociedad del Conocimiento y, en consecuencia, abordar el desarrollo de los siguientes ámbitos:

- Programas para el desarrollo de infraestructuras (banda ancha, TV digital).

- Difusión y dinamización de la sociedad de la información.

- Igualdad de género en sociedad de la información.

- Programas de eInclusión para personas con necesidades específicas (discapacidad, mayores, mundo rural).

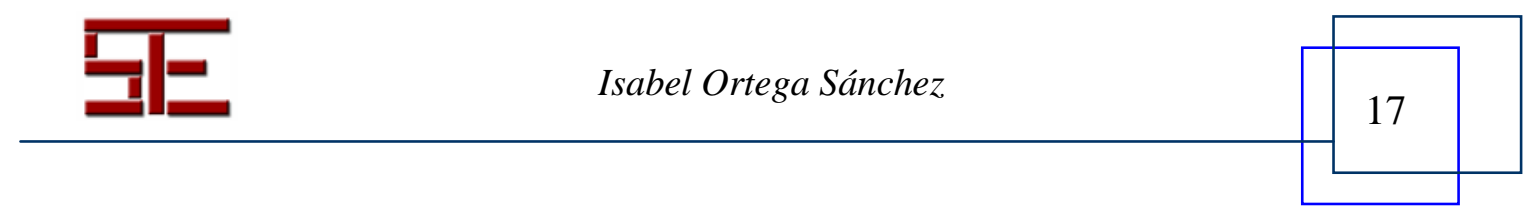




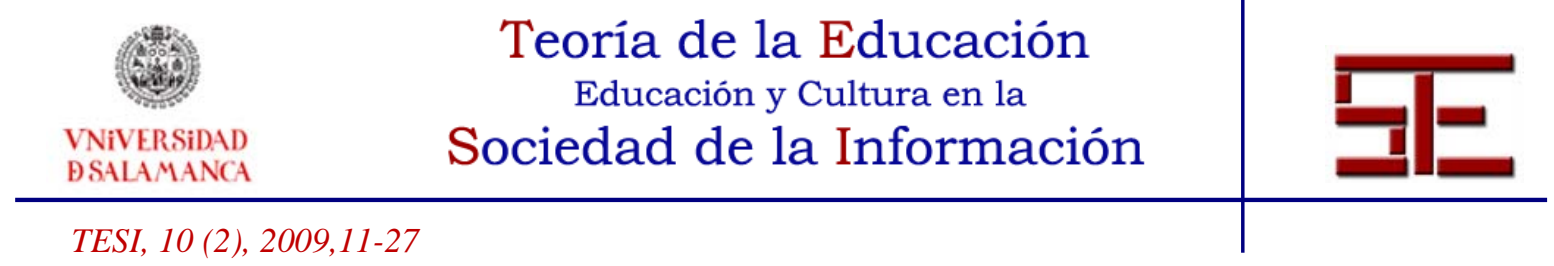

En el plan AVANZA (2005), entre las líneas desarrollada dentro de sus áreas de actuación, está la ciudadanía digital para hacer partícipes a los ciudadanos de las ventajas de utilizar Internet y las tecnologías de la información y comunicación. Uno de sus objetivos es garantizar la inclusión y el acceso a la información y la participación del ciudadano para mejorar la calidad de vida.

Destacamos entres sus numerosas líneas de actuación el programa “Avanza Ciudadanía” centrado en conseguir que las personas con necesidades especiales se incorporen a la sociedad de la información mediante actuaciones adaptadas a cada uno de los colectivos que integran la brecha digital: Accesibilidad y mayores, políticas e igualdad de género, etc.

Los avances tecnológicos tienen que desarrollar la incorporación de las personas a la sociedad y no la exclusión, desarrollando un diseño para todos y alcanzar la integración social de los colectivos excluidos sin e-accesibilidad: discapacitados, mujeres, adultos y personas mayores...

A nivel nacional e iberoamericano proyectos españoles como el Aula Mentor, desde el año 1993 hasta la actualidad, ha desarrollado cursos de formación online que capacita a los alumnos en conocimientos específicos de un área concreta y alfabetiza tecnológicamente. Atiende a la población situadas en áreas rurales facilitando el acceso a la formación, al conocimiento.

El Proyecto Mentor surge por la necesidad de formación que exige la población adulta al no tener la posibilidad de acceder a una formación telemática y a distancia por otros medios. El Ministerio de Educación y Ciencia, a través del Programa de Nuevas Tecnologías de la Información y de la Comunicación puso en marcha este proyecto basado en la enseñanza a través de las tecnologías de la información y la comunicación.

Dentro de la educación ofertada por el Aula Mentor encontramos una contextualización de la enseñanza que pretende la estimulación de la población adulta mediante motivaciones educativas, formativas, ocupacionales, sociales y personales concretas, con la posibilidad de adquirir conocimientos sobre nuevas tecnologías, y a través de ellas, según su ritmo de aprendizaje y desde cualquier lugar donde se encuentren.

Ortega Sánchez (2004) en una investigación realizada sobre el impacto de las aulas mentor en Castilla y León se detallaron los motivos para acceder a este tipo de

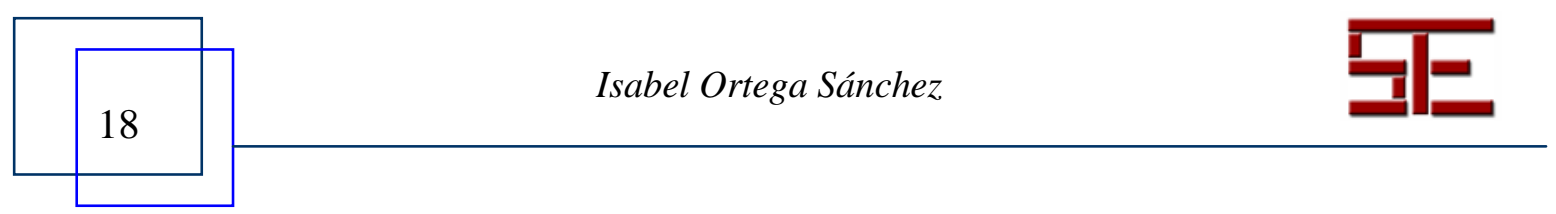




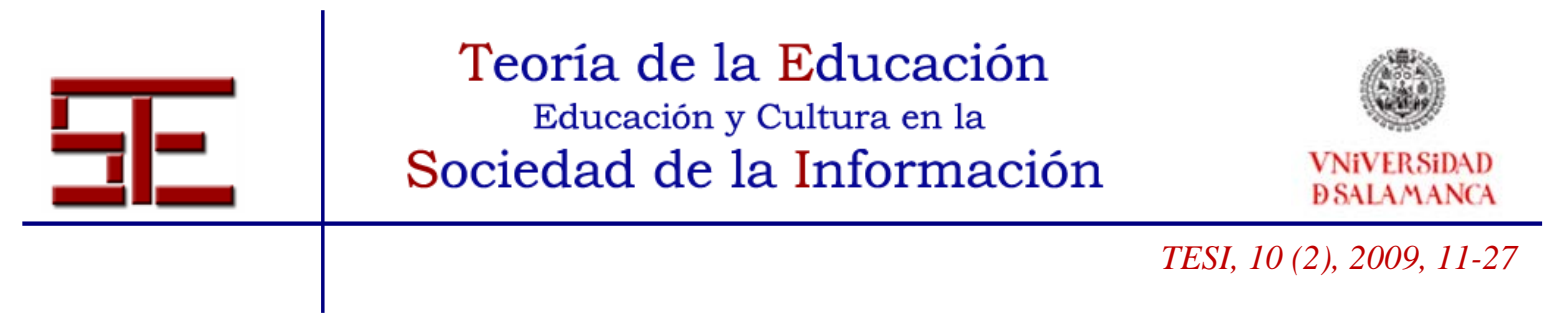

formación virtual. Los adultos resaltaban la necesidad de formar parte de la sociedad y desarrollarse personalmente para no sentirse excluidos.

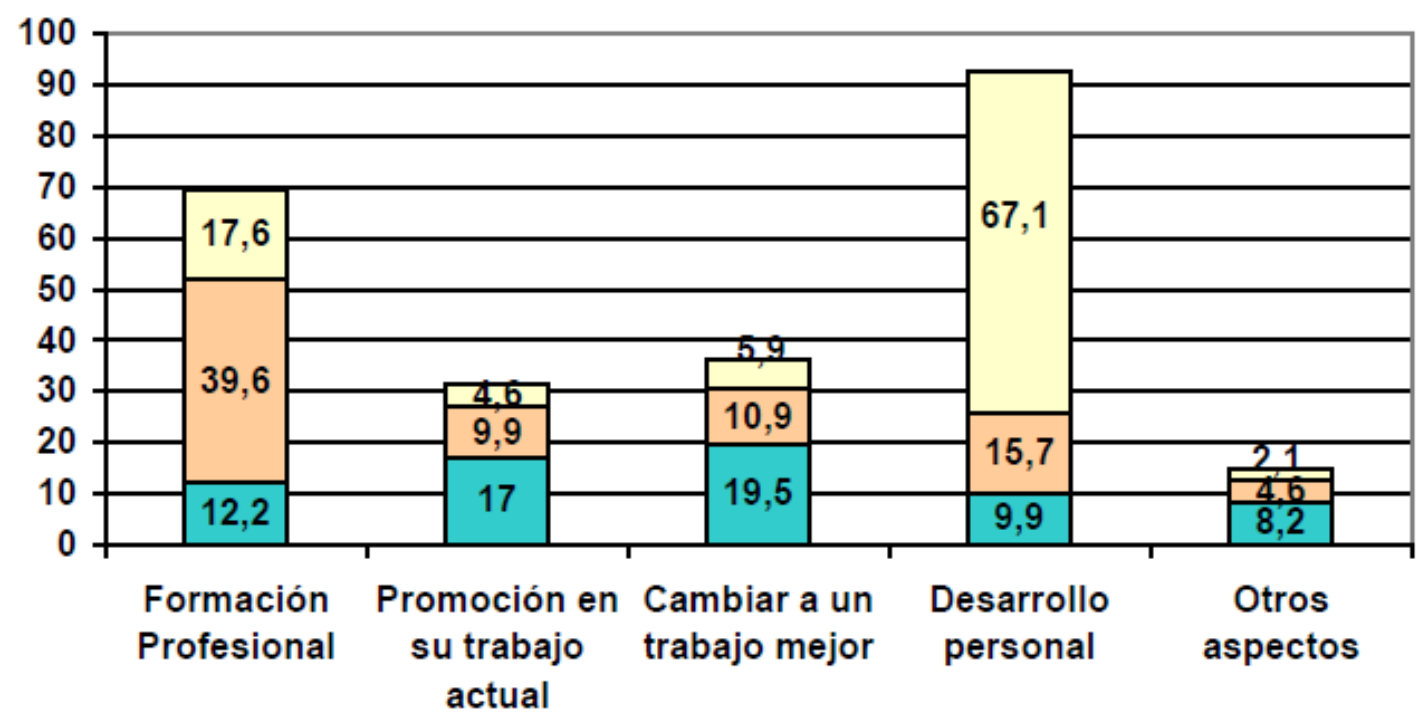

Gráfico $n^{\circ} 1$. Motivaciones para acceder a la aulas Mentor

Andalucía con el proyecto Guadalinfo extiende la alfabetización digital con énfasis en las zonas más desfavorecidas. Mirman Castillo (2006:107) opina que "Un centro Guadalinfo es también un lugar de transformación social en el que la dinamización y la movilización de los ciudadanos es la clave”

En Extremadura son innovadores en los procesos de alfabetización digital, "el proyecto de acceso a la sociedad de la información está apoyado por la conectividad y la alfabetización tecnológica. El Plan de Alfabetización Tecnológica y Software libre de Extremadura es "una acción estratégica que ha venido provocando el acercamiento y la capacitación en el uso de las tecnologías a la población adulta, especialmente a la más desfavorecida (inmigrantes, mayores, discapacitados, jóvenes, mujeres con riesgo de exclusión social)” Salado Sánchez (2006: 113)

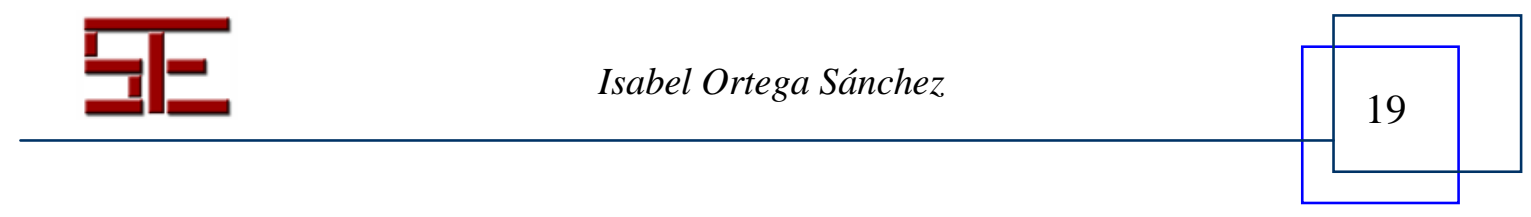




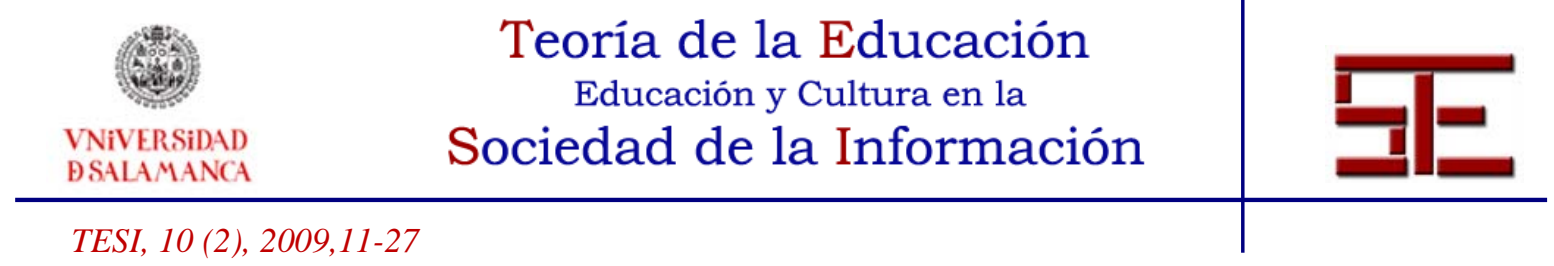

\section{LA ALFABETIZACIÓN TECNOLÓGICA EN EUROPA}

En el Consejo Europeo celebrado en Lisboa en marzo de 2000, tuvo como objetivo convertir Europa en "la economía del conocimiento más competitiva y dinámica del mundo, capaz de un crecimiento económico sostenido con más y mejores puestos de trabajo y una mayor cohesión social“. La utilización de las tecnologías ayudará al desarrollo de una sociedad competitiva y preparada para los continuos cambios económicos, sociales y profesionales.

La Constitución Europea (29 de octubre de 2004) declara que "toda persona tiene derecho a la educación y al acceso a la formación profesional y permanente”. Con la utilización de las tecnologías es posible el acceso a la formación a lo largo de la vida adquiriendo las competencias, las destrezas, las habilidades, y aptitudes cívicas, sociales y laborales para la inserción en la sociedad.

El Consejo Europeo estableció el año 2005 como el "Año Europeo de la ciudadanía” a través de la educación que juega un papel crucial en el desarrollo de la ciudadanía y a favor de la participación en la sociedad democrática, animando a los Estados miembros a la puesta en práctica de políticas de Educación para la Ciudadanía Democrática (ECD) y Educación para los Derechos Humanos (EDH).

El acceso al conocimiento, a la educación, es factor de inclusión social, de participación laboral y ciudadana. Las tecnologías de la información y la comunicación nos abren un camino hacia la democratización de la educación, posibilitando el aprendizaje de los ciudadanos. A nivel internacional existe una enorme desigualdad en las condiciones socioeconómicas y en el acceso a la educación y a las tecnologías. La educación a través de las tecnologías debe atender de manera prioritaria a los sectores de población excluidos con la finalidad de reducir las brechas educativas y digitales.

El proyecto eAbilites desarrolló la implementación de acciones de investigación, educación y transferencia de tecnología en el campo de la accesibilidad de las TIC en Europa. Según la recomendación Europea de 2006 sobre desarrollo de competencias básicas, la competencia digital es importante debido al sistema productivo actual y la demanda nuevas cualificaciones y competencias profesionales y el sistema educativo deben adaptarse a ellas.

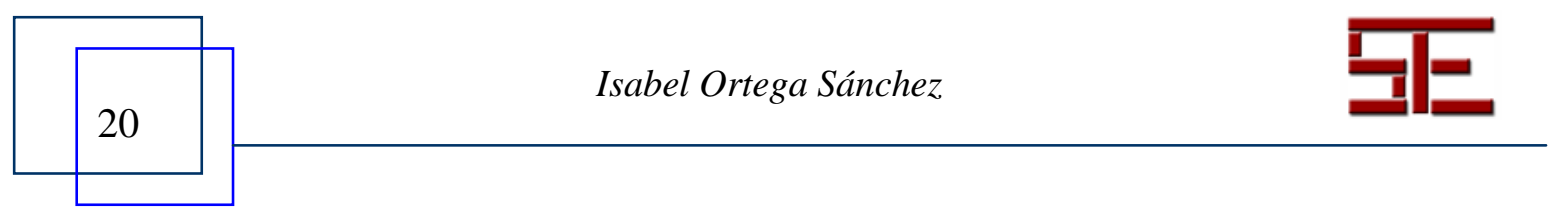




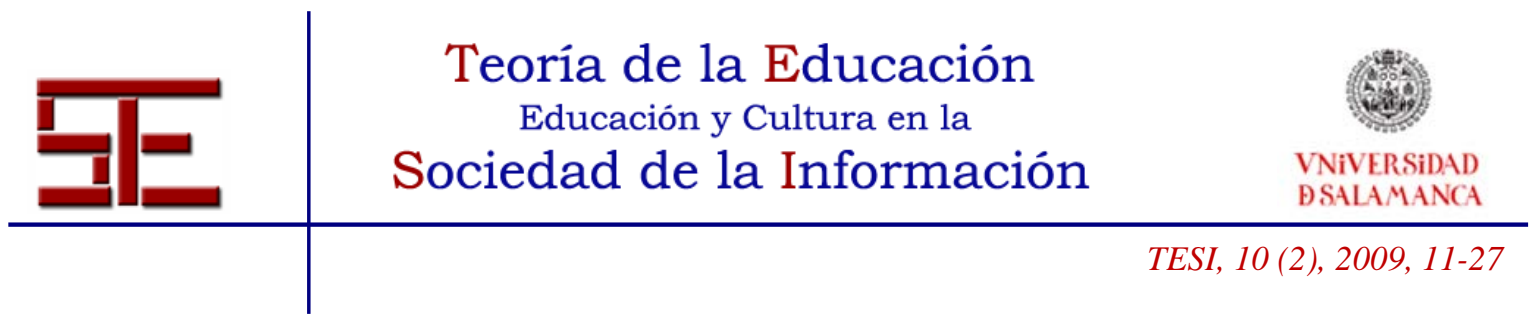

La iniciativa e-Learning ha sido hasta diciembre de 2006, el instrumento que se centró en el desarrollo de la utilización de las TIC en el aprendizaje permanente. Los Programas Marco han financiado la investigación sobre el uso de las TIC para el aprendizaje como parte del Programa de la Sociedad de la Información.

El programa Contentplus (2005-2008), trata de responder a la necesidad de desarrollar contenidos digitales de calidad para la educación, más accesibles y utilizables.

Actualmente el Programa Marco de Competitividad e Innovación (CIP) (2007-2013), paralelo al VII Programa Marco, promoverá la adopción de TIC en las empresas, la administración y los servicios del sector público.

En el 2008 se propone una Iniciativa Europea de e-Inclusión usando la información y las tecnologías de comunicación para ayudar a los ciudadanos a superar las desventajas derivadas de su situación económica, social, educacional, territorial o de alguna minusvalía. Los objetivos de e-Inclusion son; salvar la brecha en el uso de Internet en grupos en riesgo de exclusión, mejorar la cobertura de banda ancha a por lo menos el 90\% del territorio europeo y hacer todos los sitios públicos accesibles para el 2010.

La Unión Europea debe fomentar las actuaciones de los Estados miembros sobre la Accesibilidad de las Nuevas Tecnologías y los Servicios Digitales ofrecidos a todos los ciudadanos incluidos a las personas con incapacidades o con dificultades para acceder a dichos medios.

La comisión europea en el año 2008, "El uso de las TIC como herramienta de innovación y hacia el aprendizaje permanente”, informa sobre la forma en que el uso del elearning se ha desarrollado en Europa desde la Estrategia de Lisboa.

Son tres las conclusiones que se expresan en este documento:

1. El impacto de las TIC en la educación y la formación no ha sido tan grande como se había previsto.

2. La incorporación de las TIC en los sistemas de educación y formación requieren más cambios por lo que respecta a los entornos de enseñanza y aprendizaje tecnológicos y organizativos en las aulas, los lugares de trabajo y en el aprendizaje informal;

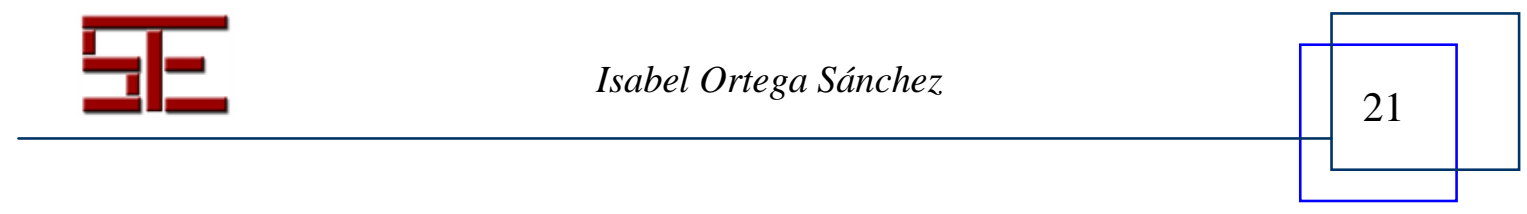




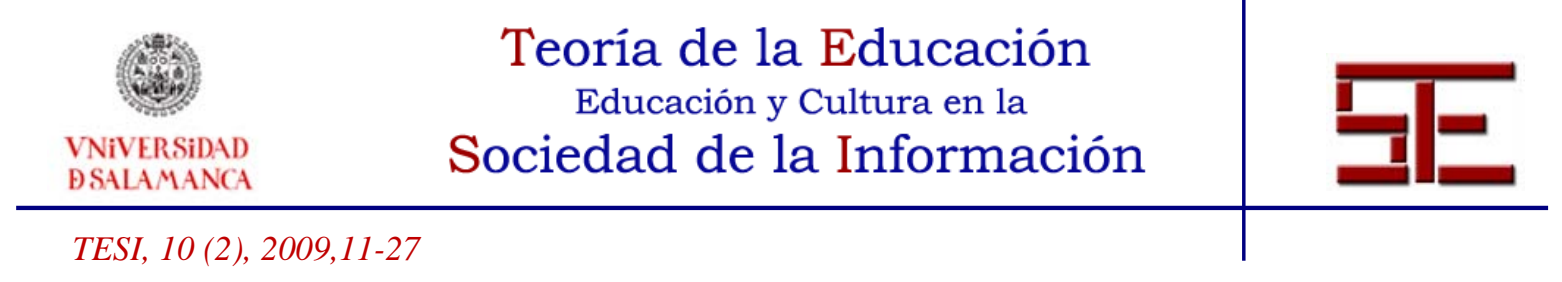

3. Las tecnologías tienen gran potencial para el desarrollo del "aprendizaje continuo" que ayude a la implantación del aprendizaje permanente formal, informal y en el ámbito laboral, aunque este hecho todavía no se ha materializado.

Las tecnologías de la información y la comunicación están beneficiando a los procesos de enseñanza-aprendizaje, en la educación superior, pero no se ha desarrollado su potencial como instrumento eficaz para el aprendizaje a distancia, la movilidad virtual, el desarrollo profesional y el aprendizaje permanente. Quizás porque aún no se han desarrollados procesos de alfabetización tecnológica que generen una enseñanza de calidad.

La alfabetización digital es imprescindible para el desarrollo profesional, el ocio y desarrollo personal, clave para el aprendizaje en general y básica para la educación y formación a través de las tecnologías.

El programa europeo "Educación y formación para el 2010” demanda a los gobiernos europeos acciones claras para mejorar los resultados educativos y así poder reducir la brecha entre el nivel de cualificación de los europeos, facilitando el acceso a las competencias claves y estimulando las capacidades para hacer frente a la sociedad del conocimiento.

i2010, la sociedad de la información y los medios de comunicación al servicio del crecimiento y el empleo, es el nuevo marco estratégico de la Comisión Europea por el que se determinan las orientaciones políticas generales de la sociedad de la información y los medios de comunicación.

La Comisión europea propone tres prioridades que se deben alcanzar antes de 2010 para las políticas europeas de la sociedad de la información y los medios de comunicación:

1. La consecución de un espacio europeo único de la información;

2. el impulso de la innovación y de la inversión en el campo de la investigación en las tecnologías de la información;

3. y la comunicación (TIC), y la consecución de una sociedad de la información y los medios de comunicación basada en la inclusión.

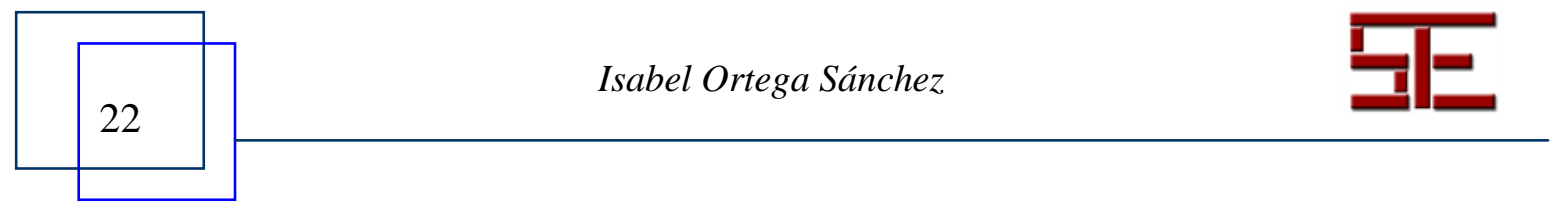




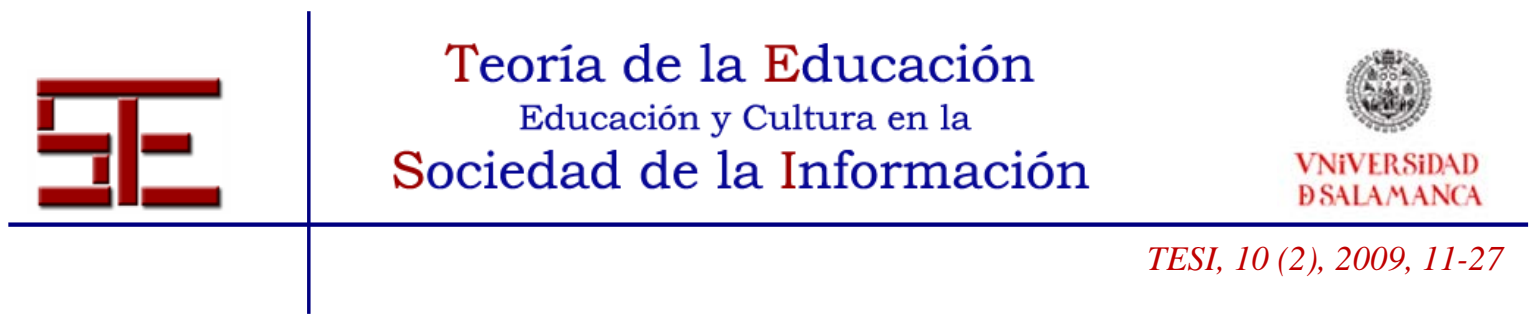

\section{CONCLUSIONES}

La alfabetización tecnológica potencia y motiva la formación virtual, ofreciendo oportunidades de aprendizaje a lo largo de la vida pero se necesitan una planificación y contextualización de las actuaciones antes de comenzar cualquier proceso de alfabetización digital. La unificación de criterios para crear una alfabetización tecnológica a nivel nacional y europeo se debe realizar y conseguir homogeneizar las competencias que desarrollen procesos de inserción social y laboral.

La alfabetización incide en la capacitación y adquisición de competencias para la mejora de la formación, la empleabilidad, el desarrollo personal y el social a través de la participación activa. Mediante la alfabetización tecnológica se consigue:

- Competencias para saber utilizar las tecnologías

- Competencias socio-comunicativas

- Se aprende a gestionar el conocimiento

- Se desarrolla el aprendizaje autónomo y el colaborativo

- Se aprenden a tomar decisiones

- Aprendizaje de nuevas formas de interacción y participación social

- Se logra una inclusión social.

- Se generan comunidades virtuales y redes sociales

- Se logra una inclusión laboral, empleabilidad

- Visión crítica de las tecnologías

- Se fomenta el aprendizaje a lo largo de la vida

Para adaptarse a la sociedad, a la vida laboral, al nuevo ocio, las nuevas formas de relacionarse e interaccionar, las redes sociales, la participación en la construcción colaborativa del conocimiento se tiene que poseer una alfabetización tecnológica generadora de todas las competencias que se demanda para formar parte de la sociedad del conocimiento.

\section{BIBLIOGRAFÍA}

Aguadez Gómez, J. I. y Pérez Rodríguez, M. A. (2006). La educación en medios de comunicación como contexto educativo en un mundo globalizado. En A. Cabero (coord.), Nuevas tecnologías aplicadas a la educación. Madrid: McGraw-Hill.

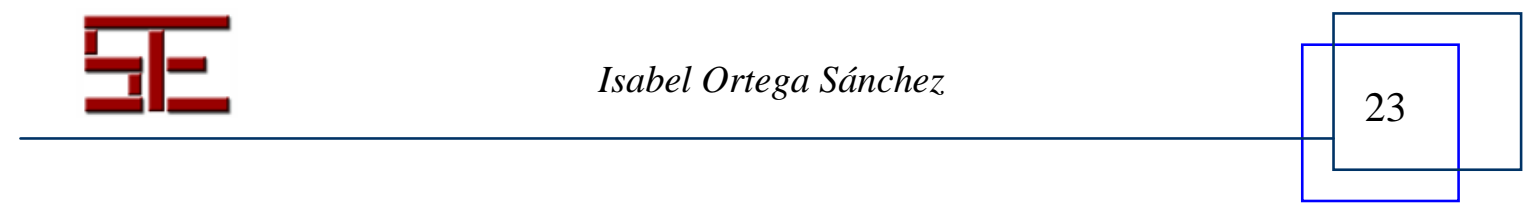




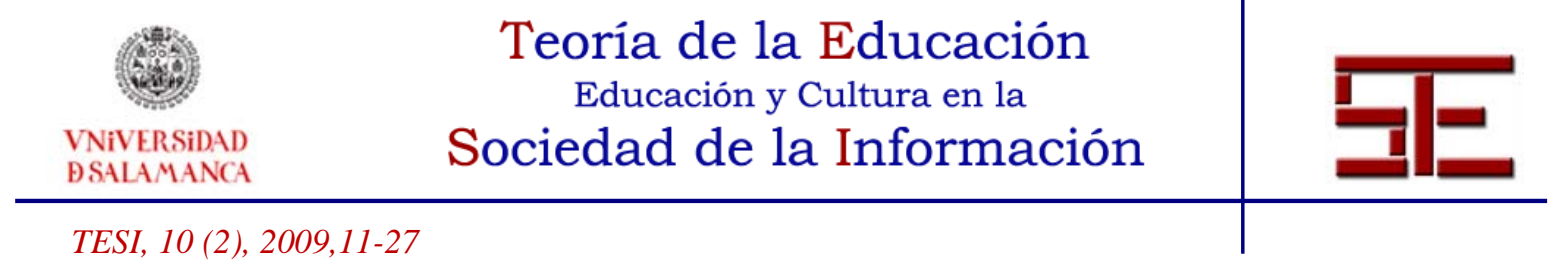

Cabero, J. (2006). Comunidades virtuales para el aprendizaje. Su utilización en la enseñanza, EDUTEC. Revista Electrónica de Tecnología Educativa, 20, [en línea]. [Fecha de consulta: 10/7/2009]. Disponible en: http://edutec.rediris.es/Revelec2/revelec20/cabero20.htm.

Casado Ortiz, R. (2006). Alfabetización tecnológica. ¿Qué es y cómo debemos entenderla? En R. Casado Ortiz (coord.), Claves de la Alfabetización digital. Barcelona: Editorial Ariel. Fundación telefónica.

Comisión Europea (2008). The use of ICT to support innovation and lifelong learning for all - A report on progress. Bruselas.

Comisión Europea (2006). Programa e-Learning. http://ec.europa.eu/education/programmes/elearning/programme_es.html (Consultado 10/07/2009).

Comisión Europea (2001). Hacer realidad un espacio europeo del aprendizaje permanente. Bruselas. 21-11-2001.

Consejo Europeo (2000). Lisboa. http://www.consilium.europa.eu/ueDocs/cms_Data/docs/pressData/es/ec/00100r1.es0.htm.

Davies, D. (2002). La competencia del aprendizaje permanente en el siglo XXI: un programa. En R. Teare, D. Davies, y E. Sandelands, Organizaciones que aprenden y formación virtual (p.92). Barcelona: Editorial Gedisa, S.A.

Delors, J. (Coord.) (1996). La educación encierra un tesoro. Informe a la UNESCO de la Comisión Internacional sobre la educación para el siglo XXI. Madrid: Santillana.

García Aretio, L. Ruiz, M. y Domínguez, D. (2007). De la educación a distancia a la educación virtual. Barcelona: Ariel.

Gutiérrez Martín, A. (2006). La alfabetización múltiple en la sociedad de la información. En CASADO ORTIZ, R. (coord.), Claves de la Alfabetización digital. Barcelona: Editorial Ariel. Fundación telefónica. i2010: la sociedad de la información y

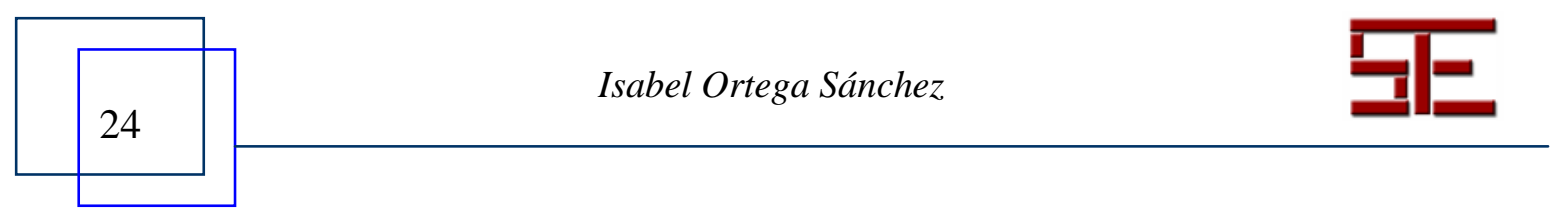




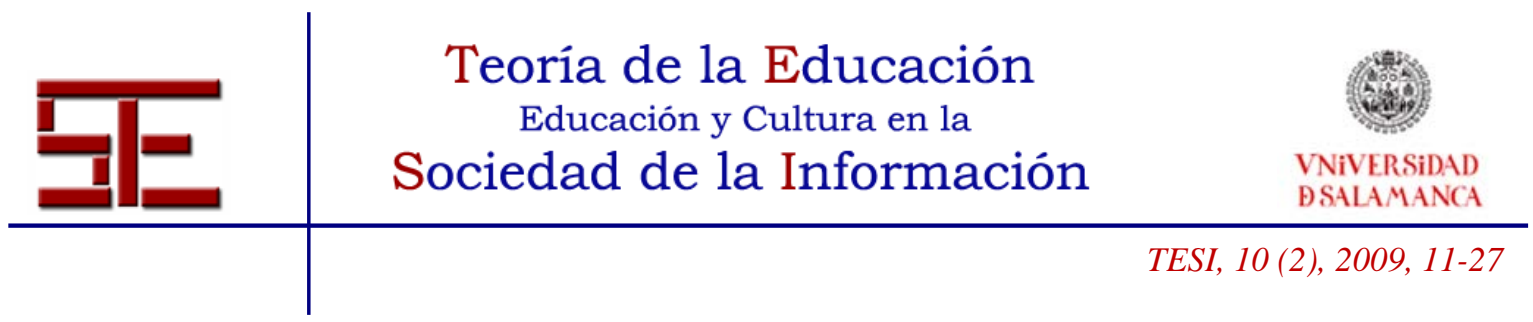

los medios de comunicación al servicio del crecimiento y el empleo http://europa.eu/legislation_summaries/employment_and_social_policy/job_creation_m easures/c11328_es.htm (Consultado 10/07/2009).

Mirman Castillo, M. (2006). Andalucía: el proyecto Guadalinfo. En R. Casado Ortiz, (coord.), Claves de la Alfabetización digital. Barcelona: Editorial Ariel. Fundación telefónica.

Ministerio De Educación Y Ciencia. Plan Nacional de I+D+I 2008-2011. http://www.plannacionalidi.es/plan-idi-public/. FECYT.

Ortega Sánchez, I. (2007). Principios organizativos y pedagógicos para el diseño de un modelo de formación virtual. En J.A. Jerónimo Montes, Aprendizaje en red, en busca de la comunidad virtual. México: UNAM, pp.151-165.

Ortega Sánchez, I. (2006). Alfabetización Tecnológica y emocional. En E. LópezBarajas Zayas, Estrategias de formación en el siglo XXI. Barcelona: Ariel.

Ortega Sánchez, I. (2004). El Aula Mentor y su influencia en la formación en la Comunidad de Castilla y León. Tesis doctoral. Madrid: UNED.

Parlamento Europeo Y El Consejo De La Unión Europea. (2006). Decisión $\mathrm{n}^{\mathrm{o}}$ 1720/2006/CE del Parlamento Europeo y del Consejo, de 15 de noviembre de 2006, por la que se establece un programa de acción en el ámbito del aprendizaje permanente [Diario Oficial L 327 de 24.11.2006].

Parlamento Europeo Y El Consejo De La Unión Europea. (2006). Recomendación del Parlamento Europeo y del Consejo, de 18 de diciembre de 2006, sobre las competencias clave para el aprendizaje permanente [Diario Oficial L 394 de 30.12.2006].

Parlamento Europeo Y Consejo De La Unión Europea. Ciudadanos con Europa (20072013). Propuesta de Decisión del Parlamento Europeo y del Consejo, de 6 de abril de 2005, por la que se establece el programa «Ciudadanos con Europa» para el periodo 2007-2013 a fin de promover la ciudadanía europea activa.

Plan Avanza. http://www.planavanza.es/ (Consultado12/07/2009).

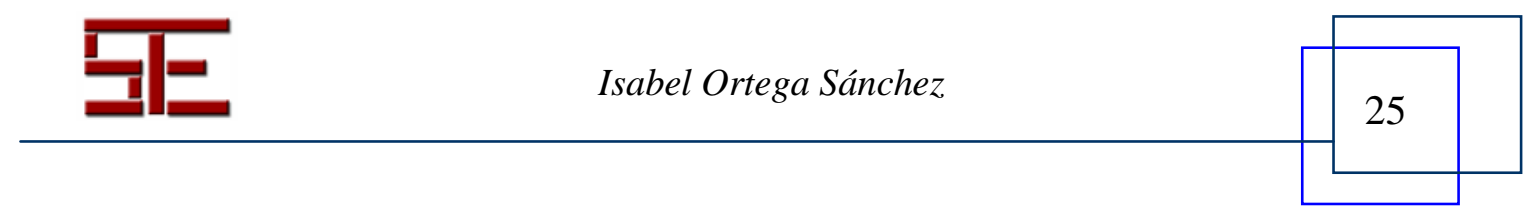




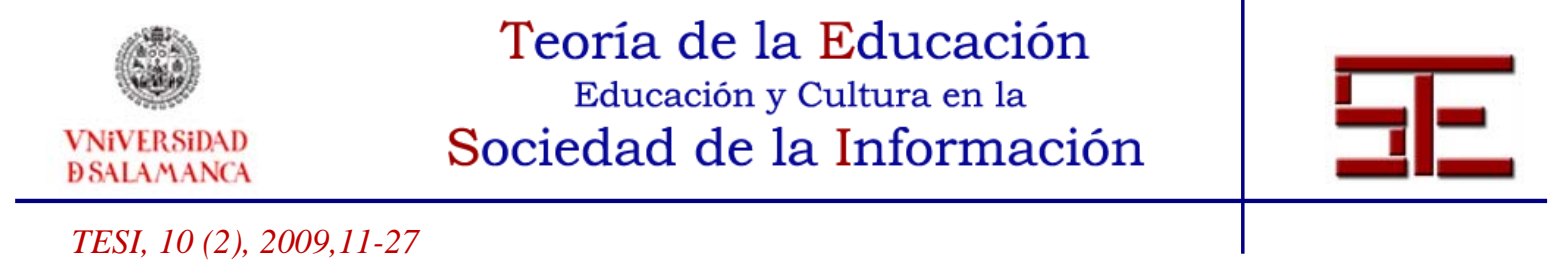

Plan De Alfabetización Tecnológica $\mathrm{Y}$ Software Libre De Extremadura.www.nccextremadura.org/ (Consultado 9/07/2009).

El Programa Econtent. http://cordis.europa.eu/econtent/ (Consultado 10/07/2009)

Programa Marco De Competitividad E Innovación (2007-2013). http://www.cordis.lu/innovation/en/policy/cip.htm (Consultado 8/07/2009).

Proyecto Eabilities. http://www.eabilities-eu.org/(Consultado 05/07/2007).

Proyecto Guadalinfo. http://www.guadalinfo.es/ (Consultado 11/07/2009).

Proyecto Mentor. https://centrovirtual.educacion.es/mentor/inicio.html (Consultado12/07/2009).

Requejo Osorio, A. (2006). "Políticas y estrategias en la Unión Europea”. En LópezBarajas Zayas, E. Estrategias de formación en el siglo XXI. Madrid: Ariel.

Roig Vila, R (2007). “Internet aplicado a la educación”. En CABERO ALMENARA, J. Nuevas tecnologías aplicadas a la educación. Madrid: McGrawHill, pp. 223-243.

Salado Sánchez, J. J. (2006). "Extremadura: de la alfabetización tecnológica y el software libre a la ciudadanía digital”. En CASADO ORTIZ, R. (coord.) Claves de la Alfabetización digital. Barcelona: Editorial Ariel. Fundación telefónica.

Sevillano García (1998). Enseñanza-aprendizaje con medios de comunicación y nuevas tecnologías. Madrid: UNED.

Trilla, J. (1990). Introducción. La Ciudad Educadora/La Ville Edcatrice, I Congrés Internacional de Ciutats Educadores (Barcelona), p. 18.

UNESCO (2008). Estándares de competencias en TIC para docentes. http://portal.unesco.org/es/ev.phpURL_ID=41553\&URL_DO=DO_TOPIC\&URL_SEC TION=201.html http://cst.unesco-ci.org/sites/projects/cst/default.aspx. (Consultado 10/07/2009).

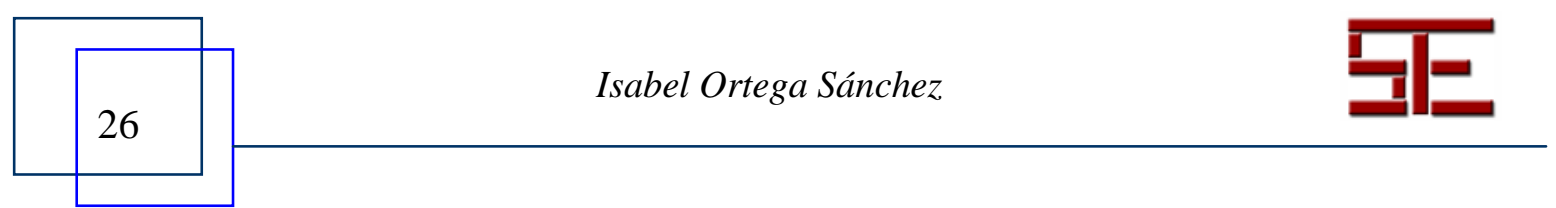




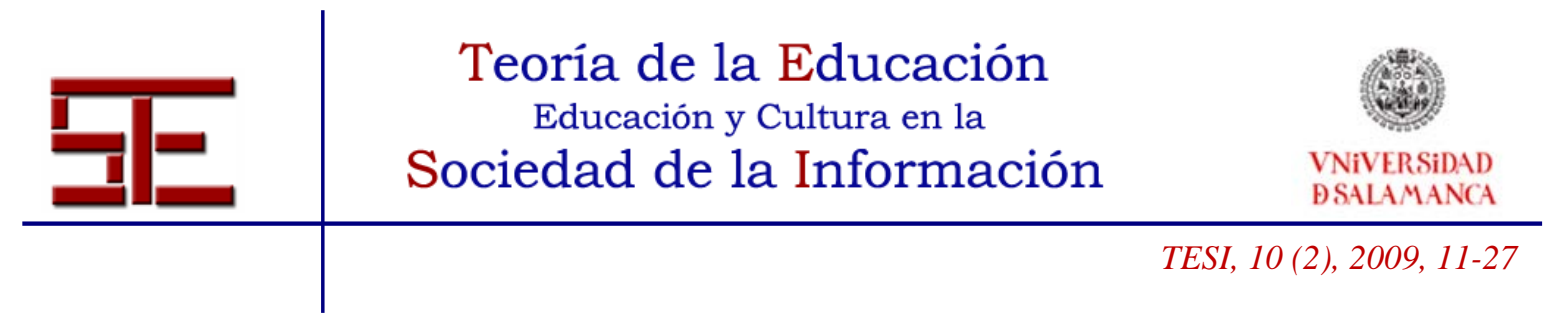

Finquelievich, S. (2005). Construyendo una Sociedad de la Información en el Sur El rol de los Gobiernos y la Sociedad Civil. Instituto del Tercer Mundo (ITeM).

Para citar la presente editorial puede utilizar la siguiente referencia:

Ortega Sánchez, I. (2009). La Alfabetización tecnológica, en Ortega Sánchez, I. y Ferrás Sexto, C. (Coord.) Alfabetización Tecnológica y el desarrollo regional [monográfico en línea]. Revista Electrónica Teoría de la Educación: Educación y Cultura en la Sociedad de la Información. Vol. 10, $\mathrm{n}^{\circ}$ 2. Universidad de Salamanca [Fecha de consulta: $\mathrm{dd} / \mathrm{mm} / \mathrm{aaaa}]$.

http://campus.usal.es/ revistas_trabajo/index.php/revistatesi/article/view/7506/7537

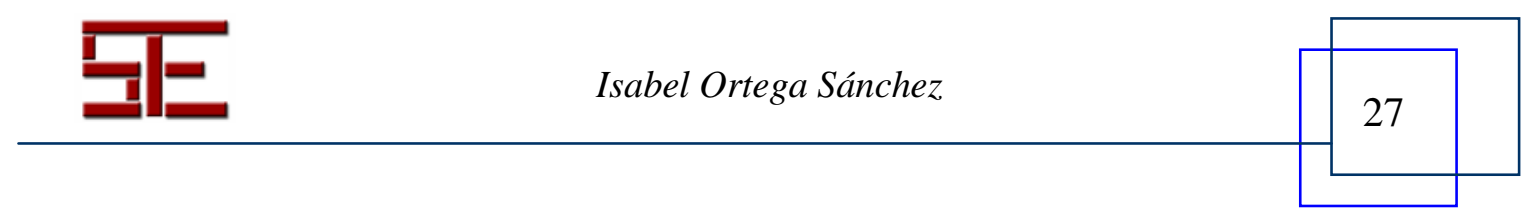

